INTERNATIONAL DESIGN CONFERENCE - DESIGN 2018

https://doi.org/10.21278/idc.2018.0303

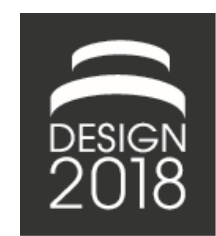

\title{
LIVE-LABS AS REAL-WORLD VALIDATION ENVIRONMENTS FOR DESIGN METHODS
}

\author{
A. Albers, B. Walter, M. Wilmsen and N. Bursac
}

\begin{abstract}
Laboratory studies and field studies make a major contribution to the validation of new design processes, methods and tools, but are confronted with limited transferability of validation results to corporate practice. In contrast, Live-Labs are validation environments that promise a high degree of external validity, but have not yet been able to systematically justify it. This article presents a procedure model and a categorisation framework that allows existing Live-Labs to be designed in such a way that the transferability of the results of Live-Lab studies to companies can be optimised.
\end{abstract}

Keywords: Live-Labs, validation environment, design research, design methods, research methodologies and methods

\section{Introduction}

In most cases just a few common design processes, methods and tools are used within the product engineering process of a company. Hence, a fundamental aspect for the successful transfer of new design processes, methods and tools is the fit and adaptability of them to the respective design situation, regarding their real-world application (Grabowski, 1997; Birkhofer et al., 2005; Geis et al., 2008). This presupposes that the research results with regard to design processes, methods and tools are, on the one hand, universally valid enough to be applied to different companies (external validity). On the other hand, it is also necessary that the research findings correctly reflect the correlations between elements of these methods, processes and tools (internal validity). But unfortunately, there often exists a tradeoff in empirical research between the internal validity, which can be achieved through laboratory studies or experiments and the external validity, which can be ensured through the conduction of various field studies or case studies (Roe and Just, 2009). To relax this harsh trade-off between internal and external validity, Live-Labs were developed as validation environments for design research (Walter et al., 2016). Due to the increasing importance of Live-Labs as validation environment for new design processes, methods and tools, this contribution provides a procedure model for the systematic design of Live-Lab environments in order to overcome the big problem of some design research programmes which lies in the fact that the respective results are being seen as largely irrelevant by companies.

\section{State of the art}

\subsection{System of objectives, operation system and system of objects}

According to the systems theory, product engineering can formally be described as the transformation of a system of objectives into a system of objects by an operation system (Albers, 2010). All objectives regarding the product engineering project are defined within the system of objectives. The system of objects includes all physical and virtual objects, which are developed within the design process and thus 
represent (partial) solutions of the respective project. The operation system is defined as socio-technical system, which consists of structured activities, design processes, methods and tools and the entire resources, which are required to transfer the system of objectives into the system of objects (Albers, 2010; Ropohl, 2012). The entirety of these three systems is also known as advanced system triple and describes the design engineer as thinking and operating individual in the centre of an uncertain and iterative product engineering process. Hence, the operation system includes as subsystems, the knowledge base and the solution space. Due to the interdependencies of the knowledge base and the solution space, it is possible to shape an iterative process model based on the processes of analysis and synthesis. Through a continuous application of analysis and synthesis activities within the operation systems, the co-evolutionary development of the system of objectives and the system of objects can be achieved (Lohmeyer, 2013; Walch, 2017). Based on the advanced system triple, a distinction between product engineering activities with a focus on design and activities with a focus on validation can be made (Albers et al., 2016a).

\subsection{Design methods}

A method describes a planned procedure to achieve a defined objective (V.D.I., 1999). Hence, methods can be specified as operative elements, which describe a procedure to solve a given problem step by step (Lindemann, 2009). Furthermore, there are different approaches in literature, which support design engineers with the situation-specific selection, adaption and application of design methods, as the Munich Model of Methods (Braun and Lindemann, 2003) or the InnoFox (Albers et al., 2014, 2015). The most important aspect for a successful selection of design methods is the fit to the respective situation of the design engineer. Thus, initially it is necessary to analyse the existing methods to identify the useful methods. Based on this, the respective company must be analysed to develop a companywide method set and finally, it is necessary to analyse the project on an operational level, to provide a project-specific method mix (Ernzer and Birkhofer, 2002).

In addition, design methods can be structured into the following elements, as shown in Figure 1 (Gerrike et al., 2017). The core idea of a method represents the basic principle or theory, the respective method is aiming for. The representation of a method stands for the system of objectives of the method application as e.g. the development of at least ten product ideas through the utility of a creativity method. The procedure of the method describes systematically the necessary actions to conduct the method and thus enables the user to apply the method. The description of the method provides the method user an explanation, as well as further information on the possible adaption and the limits of the method elements. The tool supports the user while applying the method to achieve appropriate method results. The intended use describes the scope of a method and thus gives the user an overview of the applicability of the method in respective situations within an industrial design process.

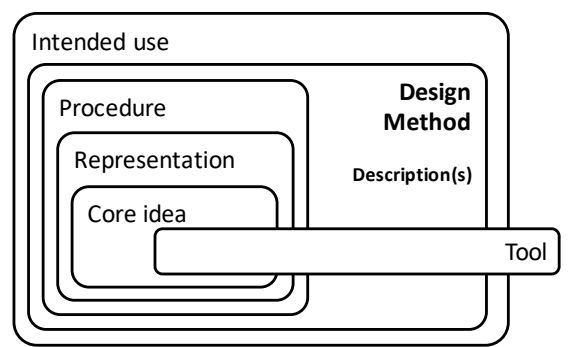

Figure 1. Elements of a method and relationship to its implementation in a tool

Based on this framework, it is possible to systematically develop new methods or method variants based on existing method elements. Furthermore, design methods elements become accessible for validation. Based on this, it is possible to validate the core idea of a new method first. This can be followed by the validation of further method elements through different validation studies, if necessary or useful. Thus, a design researcher can focus on the validation of a specific method element or the combination of various method elements and can choose the most suitable validation environment. 


\subsection{Types of scientific studies in research}

Within social sciences, there exist the distinction between descriptive research, which focuses on the observation and description of what is going on and the explanatory research, which answers the question of why something is going on (Vaus, 2001). To study complex phenomena within a real-world context, case studies or field studies are used. In general, case studies are used to answer the questions "how" and "why". Furthermore, as the behaviour of the involved persons cannot be manipulated by the researcher, it remains unclear whether an effect is caused by contextual conditions or the phenomenon itself (Yin, 2013; Baxter and Jack, 2008). In contrast to this, laboratory experiments are used to examine the cause-effect relationship and can be understood as a formally arranged intervention. Based on the comparison of different interventions, regarding the experimental conditions and the proper experimental procedure, it is possible to conclude, that the examined interdependent variable causes an effect in some aspect of the dependent variable (Boniface, 1994).

Regarding the utilization of these scientific studies as validation environment within design research, the main aspect of differentiation is the addressed type of validity. The internal validity of research results is defined as the ability to argue that the observed correlations are causal. In contrast, external validity describes the ability to abstract the observed research results to other persons and settings. Hence, laboratory experiments are useful to achieve a high level of internal validity and case studies can be utilized to gain external validity. To relax the harsh trade-off between internal and external validity, other forms of scientific studies have been introduced in science, as for example field and natural experiments (Heekerens, 2005; Roe and Just, 2009). In the context of product development, Live-Labs can be seen as the attempt to introduce an analogous approach.

\subsection{Live-Labs as validation environments for design methods, processes and tools}

In context of product engineering, Live-Labs are validation environments, which enable a design researcher to investigate design processes, methods and tools under realistic conditions and with a high controllability of the boundary conditions at the same time (Albers et al., 2016b). Examples for LiveLabs are innovation projects with companies and students (as sometimes offered by engineering institutes at universities), consultancy projects and research projects in which it is the main objective to develop a technical solution for a customer within a predefined timeframe using restricted resources and a professional project management. Within these projects, new design processes, methods and tools are applied and can be evaluated in parallel by a design researcher using accompanying, empirical studies based on suitable data collection procedures (e.g. observation, questioning, measurement and assessment of work results). By this means, it is also possible to capture procedural, personal and social aspects of the application of design processes, methods and tools. Due to the real-world project character of Live-Labs, it is, in contrast to pure laboratory studies, possible to evaluate the suitability of new design processes, methods and tools concerning their intended use in product development projects in companies to a certain degree (Walter et al., 2017a). In other words: The real-world project character of Live-Labs holds the potential to increase the external validity of research results regarding the validation of design processes, methods and tools. A decisive factor for this is that the project participants perceive one selves mainly as product engineers and less as test persons of an empirical study. This leads to a realistic behaviour of the participants which is a fact that cannot be simulated within laboratory studies. As result of this, the participants of Live-Labs experience for example a non-suitable functionality, a too low flexibility or a bad comprehensibility of the method description as disturbing for the achievement of the project objectives. Thus, the participants of a Live-Lab study evaluate the new design elements realistically and provide detailed feedback and suggestions for improvements. In contrast to field studies, Live-Lab studies offer the advantage that the boundary conditions of the application of the methods, processes or tools within the research scope are well controllable by the design researchers. This means that disturbances are controlled or completely eliminated by appropriate procedures with the objective to increase the internal validity of the research results. Ideally, the scope of the design researcher is great enough to explore several process, method or tool variants within the framework of Live-Lab studies and employ control groups. In addition, for design researchers it is usually much easier to get access to Live-Lab studies, for example by adapting existing, suitable student development projects, than to get access to corresponding field studies, which take place in companies and require a 
major intervention into development projects. Live-Lab studies can be located between laboratory studies at one side and field studies on the other side (cf. Table 1). Regarding the scope of the research results, Live-Labs can contribute to solve the conflict between the results of laboratory studies which tend to high internal validity but low external validity and the results of field studies with a tendency to high external validity but low internal validity. Compared to the findings from pure laboratory studies, the transferability of the findings from Live-Lab studies into a real-world application can therefore usually be assessed as better. In comparison to field studies, the findings from Live-Lab studies are to be assessed as more precise, because Live-Labs enable a better controllability of the boundary conditions to exclude alternative explanations regarding found effects. In addition, the results of field studies are normally very company-specific, because the design processes, methods and tools within research scope must be strongly adapted to the needs of a company to get access to real-world projects as validation environments. Hence, the research object differs from company to company in a strict sense and thus the results of different field studies can only be poorly aggregated. This effect leads to a limitation of the results of field studies as validation environment for design processes, methods and tools regarding their external validity and thus in terms of their transferability to other companies.

Table 1. Comparison of design research environments

\begin{tabular}{|c|c|c|c|c|c|}
\hline & $\begin{array}{c}\text { Researchable } \\
\text { method elements }\end{array}$ & $\begin{array}{c}\text { Controllability } \\
\text { of attributes }\end{array}$ & $\begin{array}{c}\text { Reproducibility } \\
\text { of results }\end{array}$ & $\begin{array}{c}\text { Internal validity } \\
\text { of results }\end{array}$ & $\begin{array}{c}\text { External validity } \\
\text { of results }\end{array}$ \\
\hline $\begin{array}{c}\text { Laboratory } \\
\text { study }\end{array}$ & $\begin{array}{c}\text { One method } \\
\text { element (exclusive } \\
\text { intended use) }\end{array}$ & High & High & High & Low \\
\hline $\begin{array}{c}\text { Live-Lab } \\
\text { study }\end{array}$ & $\begin{array}{c}\text { All method } \\
\text { elements }\end{array}$ & $\begin{array}{c}\text { Medium to } \\
\text { high }\end{array}$ & Medium & Medium to high & Medium to high \\
\hline Field study & $\begin{array}{c}\text { All method } \\
\text { elements }\end{array}$ & Low & Low & Low & Medium to high \\
\hline
\end{tabular}

The real added value of Live-Lab studies, the promotion of the practical use of design methods is especially accessible through an appropriate combination with well-known research formats. There are numerous synergy potentials between laboratory studies, Live-Lab studies and field studies. For example, the core idea, the representation as well as the procedure, the description or the tool of a design method can be validated with little effort as part of a pre-test or a laboratory study. On this basis, it is possible to improve the method or method elements within research scope and to examine the suitability for its intended use as part of a Live-Lab study.

\subsection{Validity of Live-Lab studies}

With regard to the external validity of the results of Live-Lab studies, a distinction can be made between person-related external validity and situation-related external validity. Person-related external validity refers to the question of the extent to which test persons can be regarded as representative of the intended later user groups (e. g. product developers) of the design processes, methods and tools examined in companies. Since Live-Labs are often not set up as a dedicated research environment, but rather existing projects (e. g. courses, research projects and consulting projects) are expanded into Live-Labs, a design researcher usually has hardly any influence on the staffing of a Live-Lab, which depends on the constraints of the respective projects (Roe and Just, 2009).

Situation-related external validity is a measure of the artificiality of Live-Lab studies. It can be understood as the total of the deviations between the application situation of a process, a method or a tool in the context of a Live-Lab study and the later application situation in companies (i. e. the intended use). Both application situations can be understood in a simplified way as an entirety of elements from the system of objectives, the operation system and the system of objects and made more comparable at this level. In order to increase the situation-related external validity, it is advisable to adapt the situation of process, method and tool applications in Live-Lab studies using these elements as closely as possible to the intended method application in companies (Roe and Just, 2009). There exist already a number of Live-Lab studies in the area of product development that have been carried out mainly in recent years. 
For example, Walter et al. (2017b) investigated creativity methods for distributed development teams, Gräßler et al. (2017) explored the application of virtual reality in early phases of product development and Reiß et al. (2017) researched the increase of method acceptance by product developers through method games. The authors of Live-Lab studies can usually argue plausibly that the design processes, methods and tools they investigate are better suited for use in companies than those that have been positively evaluated in comparable laboratory studies. However, there has been no systematic adaptation of the Live-Lab environments used to date to the intended use in companies when carrying out LiveLab studies. On the one hand, the degree of transferability of validation results into practice in companies is therefore often unclear, even regarding Live-Lab studies. On the other hand, it can be assumed that there is great potential for increasing the external validity of the results of Live-Lab studies.

\section{Research questions and research methodology}

The state of the art shows that Live-Labs are promising validation environments for the research of design processes, methods and tools which have already been able to demonstrate their added value for design research. But there still exist untapped potentials of Live-Labs, because the existing possibilities to systematically adapt Live-Labs in respect to their future real-world application of design processes, methods and tools to be investigated are not researched sufficiently, yet. Thus, the main purpose of this contribution is to promote the improvement of the significance and practical relevance of research results from Live-Lab studies. To achieve this objective, an approach shall be developed which enables design researchers to shape existing Live-Lab environments regarding the boundary conditions of the intended use of the process, method and tool within research scope in relevant industries and companies. Based on this, the approach shall enable design researchers to accurately describe and justify the scope of validation results of Live-Lab studies in detail. Therefore, the following research questions are to be answered:

- How can design researchers be supported effectively to adapt existing Live-Labs for planned Live-Lab studies in respect to the intended use of the processes, methods and tools to be investigated?

- How can the application of design processes, methods and tools in companies with specific focus on the hierarchic level of decision-makers be formalised as basis for the systematic design of Live-Lab environments?

- How can the external validity of the validation results of Live-Lab studies be accurately described and justified based on commonalities and differences between the application of design processes, methods and tools within the Live-Lab environment and their intended realworld application?

In order to answer these research questions, a research programme is defined which is based on the Design Research Methodology (Blessing and Chakrabarti, 2009) (cf. Figure 2). In descriptive study I, some of the Live-Labs (IP - Integrated Product Development, ProVIL - Product Development in a Virtual Idea Laboratory, AIL - Agile Innovation Lab (Albers et al., 2017)) existing at the IPEK - Institute of Product Development at the Karlsruhe Institute of Technology (KIT) are analysed with regard to their organizational structure and their elements using the system triple consisting of system of objectives, operation system and system of objects. In addition, the strengths and weaknesses of existing Live-Lab studies are analysed. The prescriptive study I develops a procedure model which describes the systematic design of a Live-Lab step by step. A categorization framework is being developed to formalize the application of processes, methods and tools. Based on this framework, four different cases are worked out depending on the decision-making authority of the hierarchy levels involved in the method application and influencing the method application. These cases can be used as a basis for the systematic design of a Live-Lab. Finally, the procedure model and the categorisation framework will be evaluated in a Live-Lab study using the example of the further development of an existing development method for its cross-location use (descriptive study II). 


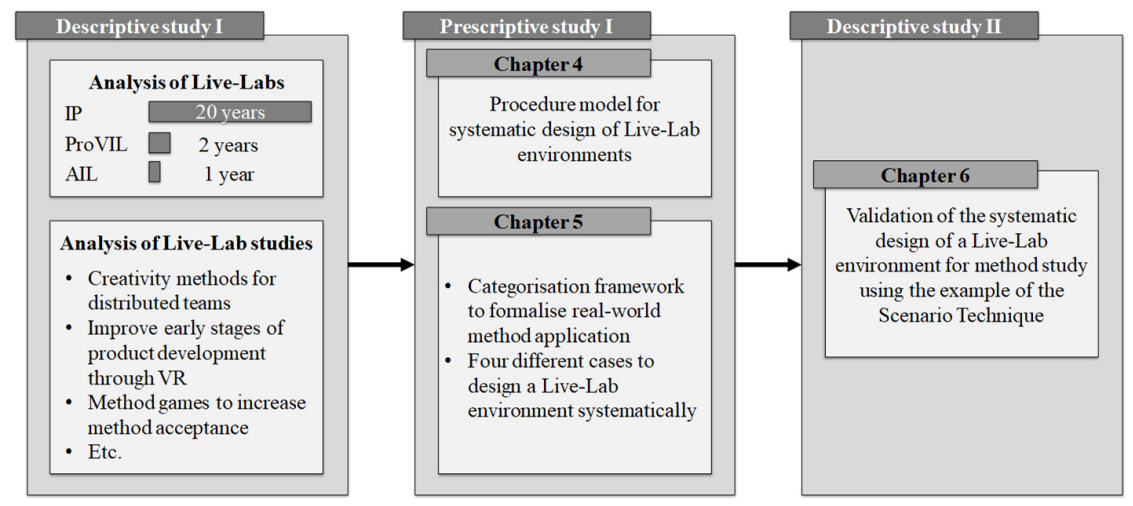

Figure 2. Overview of research methodology

\section{Procedure model for systematic design of Live-Labs}

As described within the state of the art, the intended use of a method defines the real-world circumstances and hence the boundary conditions of its real-world application. To provide a real-world validation environment for method development, it is necessary to adapt the Live-Lab environment to the boundary conditions of the real-world application as well as possible. This shall support the objective to increase the external validity and the scope of the validation results. To support this, the procedure model (cf. Figure 3) is developed.

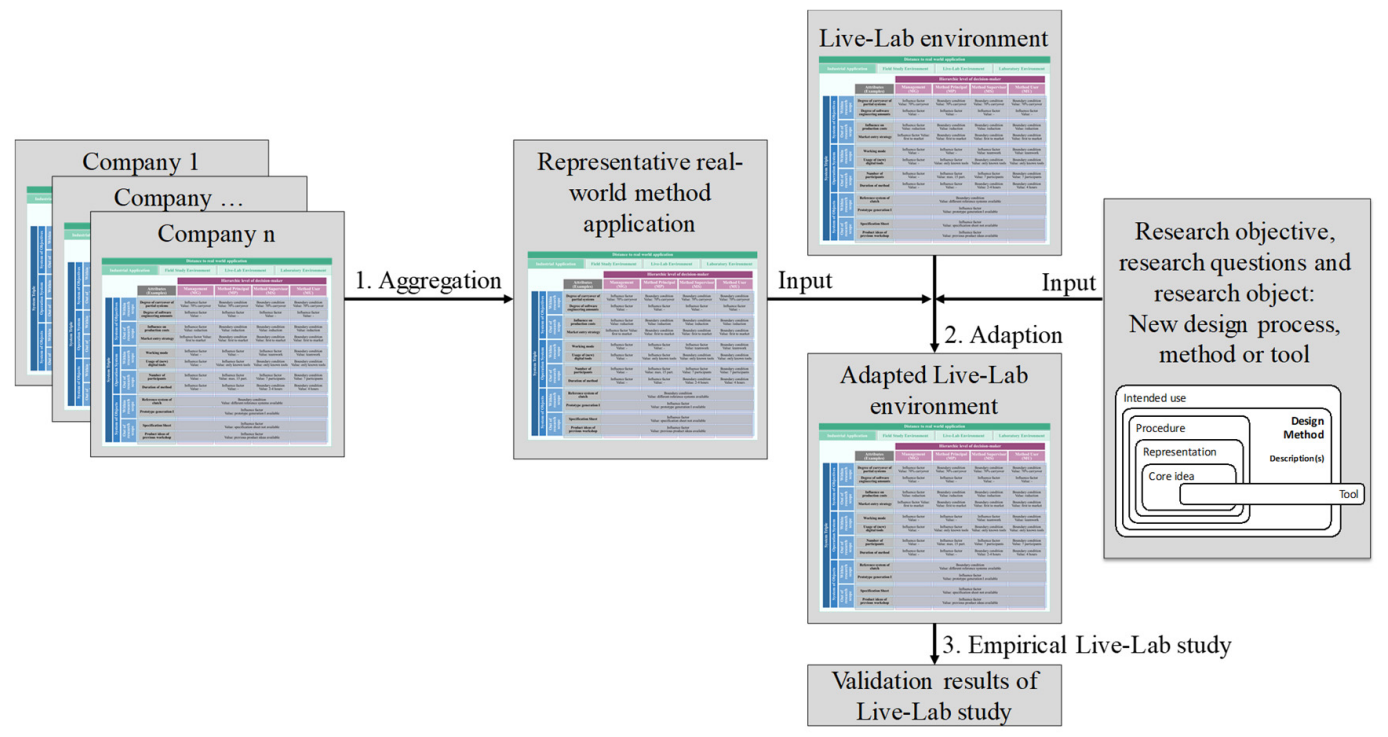

Figure 3. Procedure model for situation-specific adaption of Live-Labs

To increase transferability of validation results of Live-Lab studies, one has to know the intended use of a design process, method or tool as well as possible. As it is neither possible nor useful to develop design processes, methods and tools for a single company, the aspired scope of the process, method or tool to be investigated must be defined. This can be achieved through describing the intended use of the process, method and tool within different companies (e.g. through an interview study) and aggregation to a representative real-world method application (1. in Figure 3). In this way, for example, the usual number of participants or the normally available resources when using a method are determined. The next step (2. in Figure 3) is to customize the existing Live-Lab environment to an adapted Live-Lab environment. In this way, for example, it is ensured that the number of participants in the company's method execution corresponds as closely as possible to the number of participants in the Live-Lab. To do this, it is important to take the research objective and the research object into account as they determinate the scientific added value of adapting a specific Live-Lab attribute. As every Live-Lab 
attribute adaption represents an intervention into an existing Live-Lab project, it usually causes additional effort. Therefore, in cases where the scientific added value is low and the additional effort is high, it might make no sense to adapt the specific Live-Lab attributes. This could be the case, for example, if the usual number of participants in a method application is seven people in a company, but Live-Lab project teams consist of six people. The reshaping of the team sizes in Live-Lab to seven people might therefore improve the method validation results in terms of its external validity slightly, but could possibly cause great effort for the Live-Lab's project management. As soon as the adapted Live-Lab environment is defined, the design researcher can conduct the empirical Live-Lab study (3. in Figure 3). The Live-Lab study follows the same procedures and criteria as any comparable empirical study would do, e.g. regarding data collection, data security and statistical evaluation. The important difference to other empirical studies lies in the interpretation of the validation results (cf. Chapter 5).

\section{Systematic design of a Live-Lab environment}

The base for the systematic design of a Live-Lab environment is the identification and classification of all relevant attributes which characterise the situation of the real-world application of a design process, method or tool. An attribute describes a specific aspect of a situation and does have different possible values (e.g. 1 up to 50 project participants). As visualised in Figure 4, each attribute can be assigned to a category of the system triple including the system of objectives, operation system and system of objects. Furthermore, the system of objectives, the operation system and the system of objects can be differentiated into those attributes which are within the research scope and those which are out of the research scope. The attribute value of the application of a design process, method or tool (e.g. "eight" as value for the attribute "number of participants") is defined by a decision-maker from a specific hierarchy level (e.g. by the method supervisor) within a company.

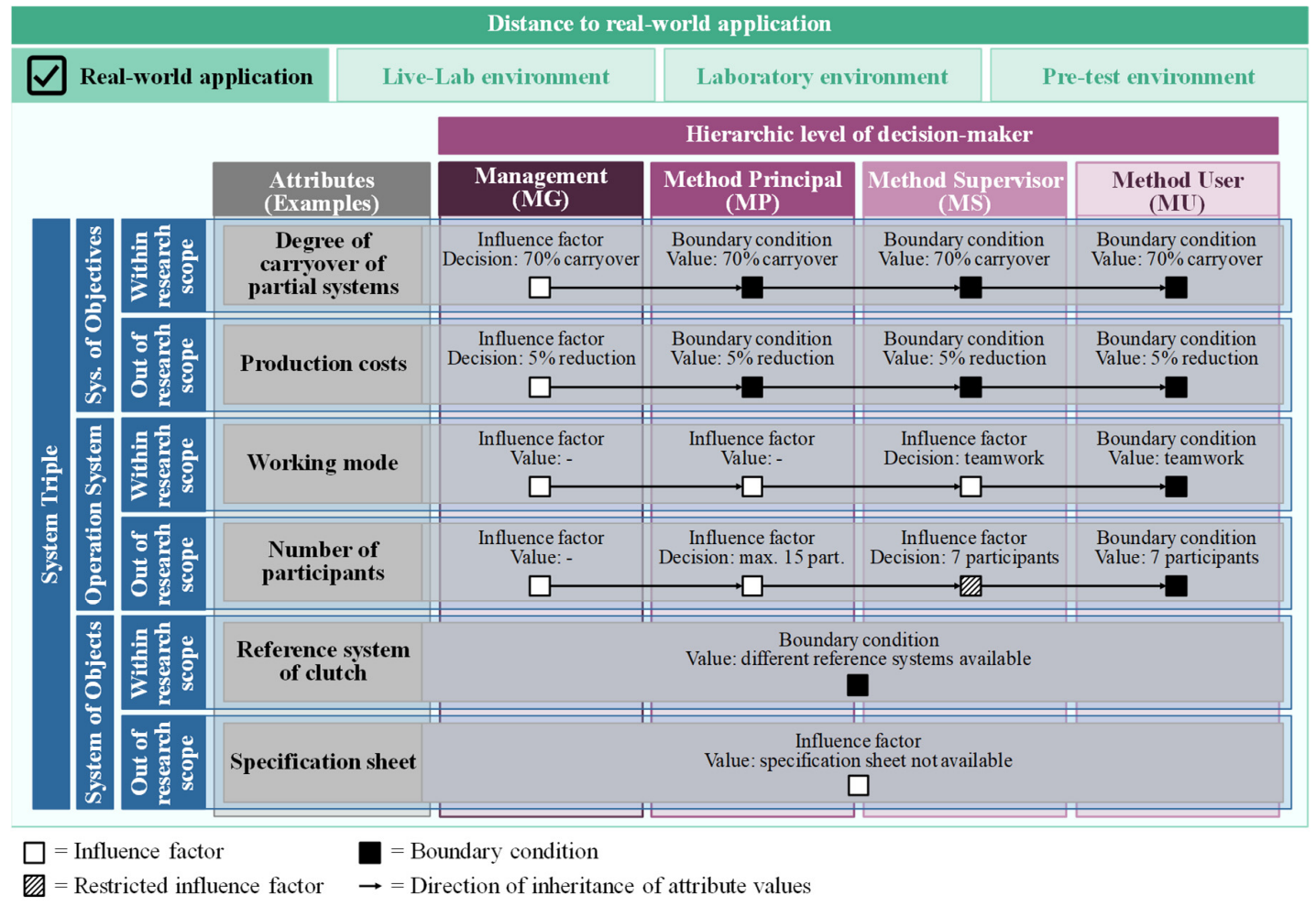

Figure 4. Categorisation framework of attributes and respective attribute values

An attribute can be a boundary condition for persons on a lower hierarchy level and an adjustable influence factor for persons on a higher hierarchy level. Thus, it is necessary to arrange the attributes depending on the hierarchy level of the decision-maker, which defines the value of an attribute. As visualised in Figure 4, the relevant categories of the hierarchy level of the decision-maker is the 
management (MG), representing the highest meaningful hierarchy level for the method application (e.g. head of department), the method principal (MP) as the entity which orders the conduction of a design method (e.g. team leader), the method supervisor (MS) as commissioned individual, who coordinates and manages the design method (e.g. a suitable team member or an external method expert) and the method user (MU) as lowest hierarchy level, which actually applies the design process, method or tool. Figure 4 shows a possible real-world application of a creativity method to develop a new clutch system. For example, the attribute in the first line represents the "degree of carryover of partial systems" and is classified as attribute of the system of objectives. Furthermore, this attribute is within the research scope of the planned Live-Lab study of the method. In this example, the management defines as objective, that the degree of carryover parts must be $70 \%$, thus the value of the attribute is " $70 \%$ carryover". Therefore, this attribute value becomes a boundary condition for all lower hierarchy levels as for the method principal, the method supervisor and the method user. Through the different combinations of boundary conditions, influence factors and respective attribute values on the side of the real-world application and on the side of the Live-Lab environment, regarding the attributes within research scope and out of scope, four different cases for the systematic design of the Live-Lab attribute values can be differentiated. These cases are shown in Figure 5 in a simplified representation. The simplification is possible, because the cases are independent of the system (system of objectives, operation system and system of objects) and of the hierarchy level, whereby the adjustment possibilities of the attribute values on level of the method users (MU) are excluded in this contribution.

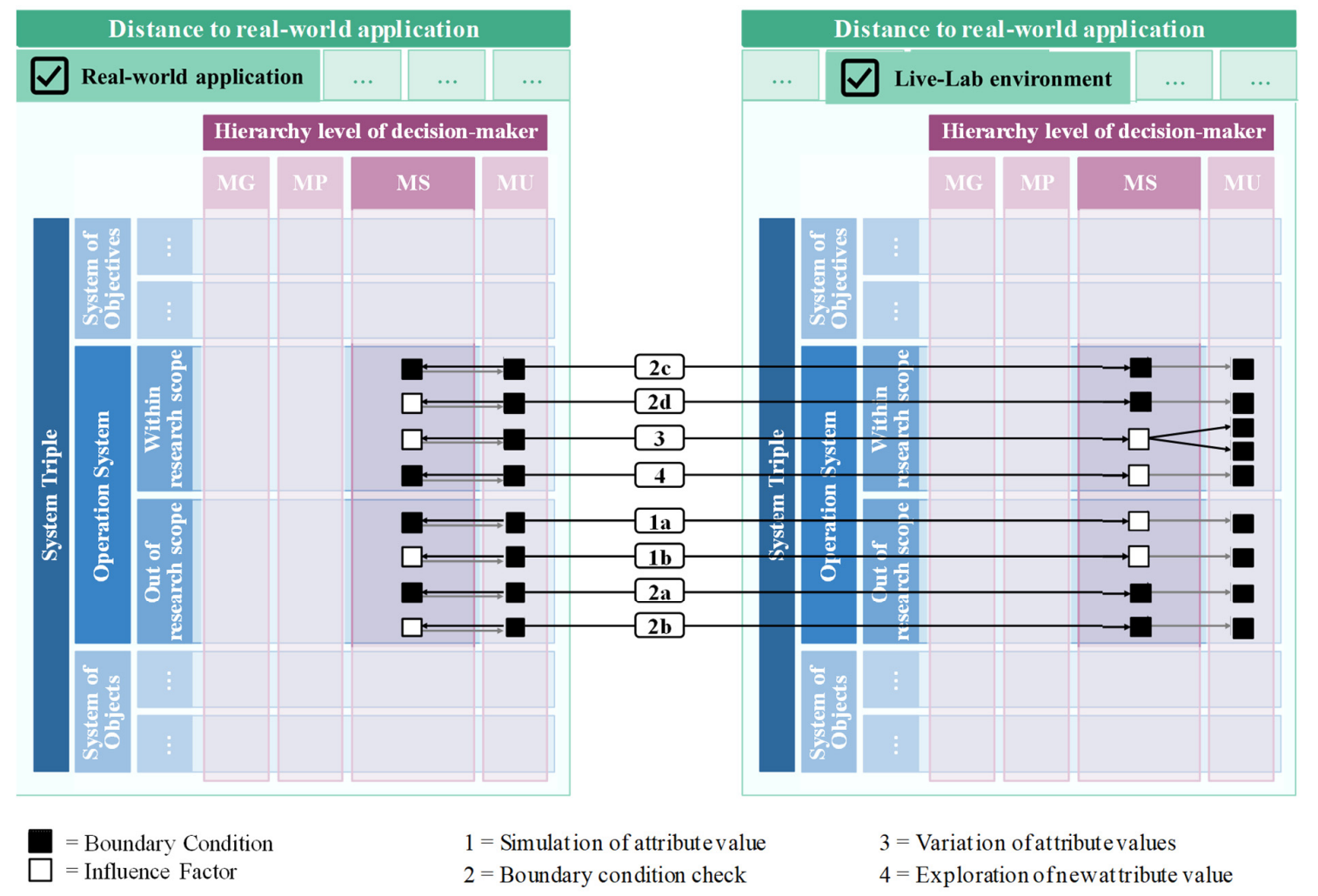

Figure 5. Simplified representation of the four different cases which are used to design a Live-Lab environment

Case 1: Simulation of attribute value: In case an attribute is out of the research scope of the regarded Live-Lab study and the attribute is an influence factor on the side of the Live-Lab environment for a certain hierarchy level of decision-makers (e.g. MS = method supervisor), this attribute value shall be adapted to the attribute value at the equivalent hierarchy level of the real-world application. Furthermore, it is not relevant whether the attribute is a boundary condition (1a in Figure 5) or an influence factor ( $1 \mathrm{~b}$ in Figure 5) for the respective hierarchy level on the side of the real-world 
application. It is only important that in this case the attribute value within the Live-Lab environment is adjusted according to the attribute value of the real-world application to simulate the method application of the real world (intended use of a method) within the Live-Lab in the best possible manner to increase the external validity and thus the scope of the validation results.

Case 2: Boundary condition check: This case becomes relevant if an attribute is a boundary condition within the Live-Lab environment and cannot be influenced by any hierarchy level. It makes no difference, whether the attribute is within the research scope (2c and 2d in Figure 5) or out of scope (2a and $2 \mathrm{~b}$ in Figure 5) or whether the attribute is a boundary condition or an influence factor on the respective hierarchy level of the real-world application. If this case occurs the two values of the attribute (within Live-Lab and for real-world application) need to be compared and analysed whether they are equal or not. As the values are the same they have a positive effect on the external validity of the LiveLab study. In case the values differ, the difference restricts the external validity of the validation results. Case 3: Variation of alternative attribute values: In case the decision-makers on the same hierarchy level on the side of the real-world application and on the side of the Live-Lab can influence the same attribute and this attribute is within research scope at the same time ( 3 in Figure 5), the attribute value can be varied in the Live-Lab. This variation means that e.g. the method supervisor of the Live-Lab develops two or more different method variants in order to evaluate two or more possible attribute values within a Live-Lab study. Based on the results of this validation study, it is possible to provide a decision support for the respective decision-maker of the real-world application (e.g. which method variant to choose) to make the conduction of the method as successful as possible.

Case 4: Exploration of new attribute value: This case can occur if an attribute is within the research scope of the Live-Lab study and the attribute value is a boundary condition for the decision-maker of the real-world application, while the respective decision-maker of the Live-Lab can decide upon the value of the attribute (4 in Figure 5). The objective of this case is to evaluate new attribute values as e.g. the usage of virtual reality tools within a Live-Lab study to increase the success of a method.

For the systematic design of the Live-Lab environment, it is important that most attributes are simulated (case 1: simulation) to ensure a sufficient external validity of the results of the Live-Lab study. Furthermore, the non-adaptable attributes need to be analysed and compared (case 2: boundary condition check) to define the scope of the validation results. To research new processes, methods or tools, only a few attributes should be varied (case 3: variation), to provide a decision support for the real-world application or explored (case 4: exploration), to demonstrate potentials of new method elements or technologies to the industry.

\section{Validation of systematic design of a Live-Lab environment}

To validate the systematic design of a Live-Lab environment, a method study has been conducted, which follows the described procedure model for a systematic design of the Live-Lab environment to increase the external validity of the validation results. The objective of the method study was the development and validation of two method variants for the application of the scenario technique for distributed product development (Walter et al., 2017c).

Initial situation: The basis for the method study was the scenario technique for the location-bound application. The scenario technique is an established method in the field of future management which enables the systematic and methodical development of various, consistent future scenarios (Fink and Siebe, 2006). Hence, the research objective was the application of the scenario technique within distributed design teams. As validation environment, the Live-Lab ProVIL - Product development in a Virtual Idea Laboratory 2017 was chosen. This course is offered and conducted annually as project for mechanical engineering master students and takes three months. In ProVIL 2017, 48 students worked on a practical engineering task provided by an industrial company with the objective to develop technically feasible solutions with high innovation potential (Walter et al., 2016).

Procedure: Based on the conduction of six interviews with experts in the field of scenario technique and distributed product engineering an aggregated real-world application was generated (1. in Figure 3 ). An extract of the representative real-world application is described within Figure 6 on the left side. Furthermore, it was possible to design the Live-Lab environment systematically (Figure 6, right), based 
on the synthesis of the developed method variants, the research objective, as well as the aggregated realworld application (Figure 6, left) and the given Live-Lab environment (2. in Figure 3).

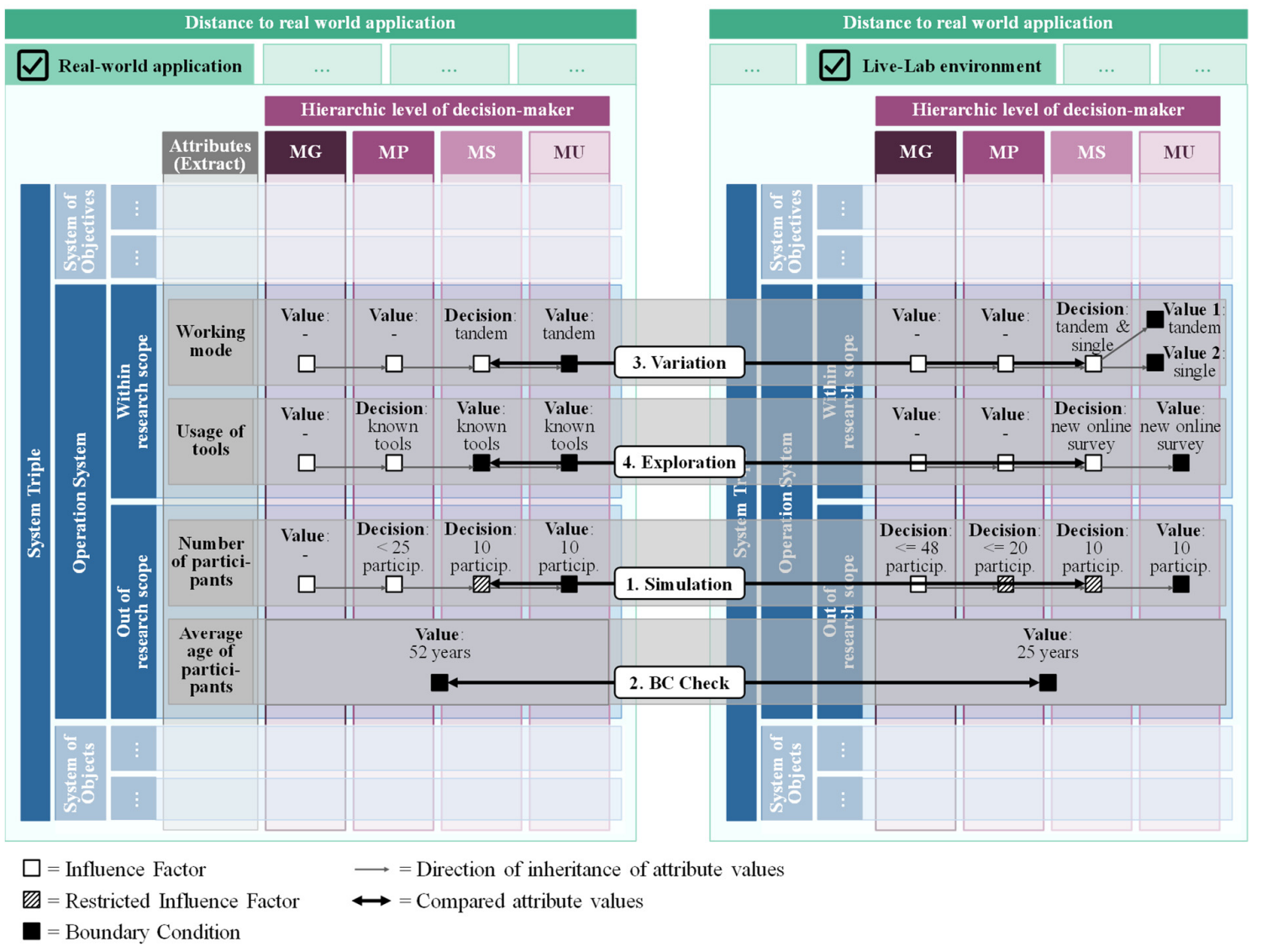

Figure 6. Systematic design of the attributes of a Live-Lab environment (extract)

Systematic design of Live-Lab environment (in respect to Figure 5 and 2. in Figure 3): To design the Live-Lab environment systematically it was important to classify the attributes regarding their belonging to the system triple and whether they are within the research scope of the Live-Lab study. As shown in Figure 6, the attributes working mode and usage of tools are within the research scope and the attributes number of participants and average age of participants are out of scope. In accordance to the already described four cases to design a Live-Lab environment (chapter 5 and 2. in Figure 3), the number of participants, with the value "10 participants" was simulated within the Live-Lab environment, to increase the external validity of the validation results. The attribute average age of participants could not be influenced neither within the real-world application, nor within the Live-Lab environment, hence the case boundary condition check was occurred. Through the discrepancy of the respective attribute values ("52 years" within real-world application vs. "25 years" within Live-Lab environment), the external validity of the validation results was negatively influenced. The attribute working mode was within the research scope of the method study and was an influence factor for the method supervisor (MS) within the real-world application and within the Live-Lab environment. Hence, the attribute was varied and two different attribute values, the work within a "tandem" and "single" work was investigated. Based on the validation results of the method study, it was possible to provide the method supervisor of the real-world application a recommendation regarding the decision on the attribute value. The attribute usage of tools was also within research scope of the method study and was a boundary condition for the method supervisor (MS) of the real-world application with the restriction to "use only tools, which are known within the company". In spite to this attribute value, the attribute was explored within the Live-Lab environment with the attribute value "usage of a new online survey", thus it was possible to achieve better results through the usage of the new tool than with the common tools. The 
utilization of the new online survey was rated positively through different experts, thus the exploration of the attribute value can be considered successfully (3. in Figure 3).

\section{Discussion and outlook}

The results of the studies described in this paper provide a concrete opportunity to systematically adapt existing Live-Labs to improve the transferability of validation results from Live-Lab studies to corporate practice and strengthen the role of Live-Labs as real-world validation environments. Thus, this contribution is based on the fundamental question of any empirical research whether and to what extent the respective research results can be transferred to circumstances outside the research environment. Design processes, methods and tools represent an object of investigation, for whose research the intended use in the sense of the socio-technical application context is indispensable. This poses challenges for validation environments with regard to the closeness to reality of personal and social factors such as the motivation and behaviour of study participants. In addition, the application of design processes, methods and tools in companies can usually be well delimited in terms of time, as well as formally described in terms of the objectives, use of resources and hierarchical levels of the involved groups of people, and with the help of the triple of system of objectives, operation system and system of objects. The present article makes use of this connection to develop an approach for the systematic adaptation of existing Live-Labs. The four cases proposed in chapter 5 represent a qualitative distinction between the different constellations that can occur between the application of design processes, methods and tools in Live-Labs and in companies. With this, they provide a reasonable basis for argumentation regarding the scope of validation results as well as a good basis for the targeted adaptation of Live-Labs. However, the present approach does not allow quantitative statements about effect strengths, e. g. to what extent deviating boundary conditions in Live-Labs and in companies (for example the different ages of participants) impair the transferability of validation results. To refine the results of this contribution, it is necessary to apply the procedure model and the categorisation framework for attributes during future Live-Lab studies. To increase the efficiency of this approach, it will be also important to develop a collection of the common and relevant attributes and respective values of typical Live-Lab environments as well as repetitive procedures which shall be collected as a Live-Lab handbook. Furthermore, it is necessary to generate a form to gather, analyse and aggregate the required information regarding the attribute values of the real-world application in companies in a standardised way.

\section{References}

Albers, A. (2010), "Five hypotheses about engineering processes and their consequences", Proceedings of the TMCE 2010, Ancona, April 12 - 16, 2010.

Albers, A., Behrendt, M., Klingler, S. and Matros, K. (2016a), "Verifikation und Validierung im Produktentstehungsprozess", Handbuch Produktentwicklung, Carl Hanser, München, pp. 543-571. https://doi.org/10.3139/9783446445819.019

Albers, A., Bursac, N., Heimicke, J., Walter, B. and Reiß, N. (2017), "20 years of co-creation using case based learning. An integrated approach for teaching innovation and research in Product Generation Engineering", Proceedings of the 20th ICL Conference, Springer. https://doi.org/10.1007/978-3-319-73204-6_69

Albers, A., Bursac, N., Walter, B., Hahn, C. and Schröder, J. (2016a), "ProVIL - Produktentwicklung im virtuellen Ideenlabor", Entwerfen, Entwickeln, Erleben Werkzeuge und Methoden in Produktionsentwicklung und Design - Dresden, pp. 185-198.

Albers, A., Reiß, N., Bursac, N., Urbanec, J. and Lüdcke, R. (2014), "Situation-appropriate method selection in product development process-empirical study of method application”, NordDesign 2014, Espoo.

Albers, A., Reiß, N., Bursac, N., Walter, B. and Gladysz, B. (2015), "InnoFox - Situationsspezifische Methodenempfehlung im Produktentstehungsprozess", Stuttgarter Symposium für Produktentwicklung.

Albers, A., Walter, B., Gladysz, B., Reiß, N., Dörr, M. and Hinkelmann, M. (2014), "Ansatz zur situations- und bedarfsgerechten Methodenauswahl in der Produktentstehung basierend auf dem Systemtripel aus Zielsystem, Handlungssystem und Objektsystem", 12. Gemeinsames Kolloquium Konstruktionstechnik 2014.

Baxter, P. and Jack, S. (2008), "Qualitative case study methodology: Study design and implementation for novice researchers", The qualitative report, Vol. 13 No. 4, pp. 544-559.

Birkhofer, H., Jänsch, J. and Kloberdanz, H. (2005), "An extensive and detailed view of the application of design methods and methodology in industry", Proceedings of the 15th International Conference on Engineering Design (ICED 05), Melbourne, Australia, The Design Society, Glasgow. 
Blessing, L.T.M. and Chakrabarti, A. (2009), DRM: A Design Reseach Methodology, Springer London, 2009. https://doi.org/10.1007/978-1-84882-587-1

Boniface, D.R. (1994), Experiment design and statistical methods for behavioural and social research, CRC Press.

Braun, T. and Lindemann, U. (2003), "Supporting the selection, adaptation and application of methods in product development", Proceedings of the 14th International Conference on Engineering Design (ICED 03), Stockholm, Sweden.

De Vaus, D.A. (2001), Research Design in Social Research, Sage Publications.

Ernzer, M. and Birkhofer, H. (2002), "Selecting methods for life cycle design based on the needs of a company." Proceedings of DESIGN 2002 / 7th international design conference, Dubrovnik, Croatia, 2002, The Design Society, Glasgow.

Fink, A. and Siebe, A. (2006), "Handbuch Zukunftsmanagement", Werkzeuge der strategischen Planung und Früherkennung, Bielefeld.

Geis, C., Bierhals, R., Schuster, I., Badke-Schaub, P. and Birkhofer, H. (2008), "Methods in practice-a study on requirements for development and transfer of design methods", Proceedings of DESIGN 2008 / 10th international design conference, Dubrovnik, Croatia, 2008, The Design Society, Glasgow.

Gericke, K., Eckert, C.M. and Stacey, M.K. (2017), "What do we need to say about a design method?", Proceedings of the 21st International Conference on Engineering Design (ICED 17), Vancouver, Canada.

Grabowski, H. (1997), Neue Wege zur Produktentwicklung, Raabe, Stuttgart.

Gräßler, I., Albers, A., Taplick, P., Walter, B., Klippert, M. et al. (2017), "Supporting Innovation and Creativity by Virtual Reality Technology - a White Paper", IRJET.

Heekerens, H.-P. (2005), "Vom Labor ins Feld", Psychotherapeut, Vol. 50 No. 5, pp. 357-366. https://doi.org/10.1007/s00278-005-0441-2

Lindemann, U. (2009), Methodische Entwicklung technischer Produkte: Methoden flexibel und situationsgerecht anwenden, Springer-Verlag, Berlin, Heidelberg. https://doi.org/10.1007/978-3-642-01423-9

Lohmeyer, Q. (2013), Menschzentrierte Modellierung von Produktentstehungssystemen unter besonderer Berücksichtigung der Synthese und Analyse dynamischer Zielsysteme, PhD thesis, Karlsruher Institut für Technologie

Reiß, N., Albers, A., Janke, M., Popp, F. and Bursac, N. (2017), "Das Methoden Spiel „SPALTEN Expedition”, Akzeptanzsteigerung durch Erfahrungen und Erfolgserlebnisse", 4. Stuttgarter Symposium für Produktentwicklung 2017 (SSP) : Produktentwicklung im disruptiven Umfeld, Stuttgart, Deutschland, June 28 - 29, 2017.

Roe, B.E. and Just, D.R. (2009), "Internal and external validity in economics research: Tradeoffs between experiments, field experiments, natural experiments, and field data", American Journal of Agricultural Economics, Vol. 91 No. 5, pp. 1266-1271. https://doi.org/10.1111/j.1467-8276.2009.01295.x

Ropohl, G. (2012), Allgemeine Technologie: Eine Systemtheorie der Technik, KIT Scientific Publishing. https://doi.org/10.5771/9783845269153.

VDI-Richtlinien (1999), 2223. Methodisches Entwerfen technischer Produkte, VDI Verlag, Düsseldorf.

Walch, M. (2017), Variantenentwicklung im ZHO-Modell im Kontext der PGE - Produktgenerationsentwicklung - Methode zur Entscheidungsunterstützung bei der Konzeptentwicklung in der Angebotsphase auf Basis abgeleiteter Varianten, $\mathrm{PhD}$ thesis, Karlsruher Institut für Technologie

Walter, B., Albers, A., Benesch, G. and Bursac, N. (2017a), "ProVIL-Produktentwicklung im virtuellen Ideenlabor: Anwendungs- und Implementierungsmodell eines Live-Labs", 4. Stuttgarter Symposium für Produktentwicklung 2017 (SSP): Produktentwicklung im disruptiven Umfeld, Stuttgart, Deutschland, June 28 - 29, 2017.

Walter, B., Albers, A., Haupt, F. and Bursac, N. (2016), "Produktentwicklung im virtuellen Ideenlabor Konzipierung und Implementierung eines Live-Lab", 27th Symposium Design for X, pp. 283-295.

Walter, B., Klippert, M., Kunz, M., Albers, A. and Reiß, N. (2017b), "Kreativitätsmethoden im digitalen Umfeld - Aktive Förderung von Innovationsimpulsen in standortverteilten Entwicklungsteams", 15. Gemeinsames Kolloquium Konstruktionstechnik Interdisziplinäre Produktentwicklung, Duisburg, October 5 - 6, 2017.

Walter, B., Wilmsen, M., Albers, A. and Bursac, N. (2017c), "Zukunftsmanagement in Zeiten der Digitalisierung: Die Szenario-Technik als Innovationsmethode in der standortverteilten Produktentwicklung”, Symposium für Vorausschau und Technologieplanung - SVT 2017, Berlin.

Yin, R.K. (2013), Case study research: Design and methods, Sage publications.

Albert Albers, Prof. Dr.-Ing. Dr. h. c.

Karlsruhe Institute of Technology

Kaiserstr. 10, 76131 Karlsruhe, Germany

Email: albert.albers@kit.edu 